\title{
Challenges to and Facilitators of Recruitment to an Alzheimer's Disease Clinical Trial: A Qualitative Interview Study
}

\author{
Clare Clement ${ }^{\mathrm{a}, \mathrm{b}, 1, *}$, Lucy E. Selman ${ }^{\mathrm{a}, \mathrm{b}, 1}$, Patrick G. Kehoe ${ }^{\mathrm{c}}$, Beth Howden ${ }^{\mathrm{b}}$, J. Athene Lane ${ }^{\mathrm{a}, \mathrm{b}}$ \\ and Jeremy Horwood ${ }^{\mathrm{a}, \mathrm{b}}$ \\ ${ }^{a}$ Bristol Randomised Trials Collaboration, Bristol Trials Centre, University of Bristol, Bristol, UK \\ ${ }^{\mathrm{b}}$ Population Health Sciences, Bristol Medical School, University of Bristol, Bristol, UK \\ ${ }^{\mathrm{c} D e m e n t i a}$ Research Group, Translational Health Sciences, Bristol Medical School, \\ University of Bristol, Bristol, UK
}

Accepted 8 April 2019

\begin{abstract}
.
Background: Low participation in clinical trials is a major challenge to advancing clinical Alzheimer's disease (AD) research and care. Factors influencing recruitment to AD trials are not fully understood.

Objective: To identify barriers to, and facilitators of, recruitment in a UK multi-center, secondary care AD trial (Reducing pathology in Alzheimer's Disease through Angiotensin TaRgeting (RADAR) trial) and implications for improving recruitment to AD trials.

Methods: Semi-structured qualitative telephone interviews with a purposive sample of 17 trial site staff explored the RADAR trial recruitment pathway and views and experiences of recruitment. Interviews were analyzed thematically.

Results: Diagnostic and care pathways hindered identifying patients with mild-moderate AD, with a lack of up-to-date patient records and data access problems affecting screening. Research is not routinely embedded in AD care but facilitated recruitment when it was. Clinicians' and patients' favorable view of the trial purpose facilitated recruitment, although the complexity of participant information sheets and requirement for study companion created challenges.

Conclusion: These findings have important implications for the design of future AD trials and for planning how to best interface with clinical commitments to ensure sufficient and timely recruitment. Challenges to AD trial recruitment can occur at care pathway, clinician, and patient and companion levels. Recruitment can be facilitated by: improving diagnostic processes and systems for recording and sharing patient information, embedding research into routine patient care, collaborating with a range of services to identify and approach eligible patients, training and engaging trial staff, and providing patients with clear and concise study information.
\end{abstract}

Keywords: Dementia, humans, patient care, qualitative research, randomized controlled trial, research design

\section{INTRODUCTION}

The success of randomized controlled trials (RCTs) relies on adequate and timely recruitment.

\footnotetext{
${ }^{1}$ These authors contributed equally to this work.

*Correspondence to: Clare Clement, Bristol Medical School, University of Bristol, Canynge Hall, 39 Whatley Road, Bristol, BS8 2PS, UK. Tel.: +01179 28 7272/07870 159065; E-mail: c.clement@bristol.ac.uk.
}

Common reasons for poor recruitment to RCTs include potential participants' understanding of trial processes such as randomization [1], patient and health professional preference for particular treatments [2-4], and barriers to communication across trial sites. A review of 24 multi-site phase II and III Alzheimer's disease (AD) clinical trials found that only a third were able to recruit sufficient participants within a year [5]. AD trials may face additional 
recruitment challenges due to complex medication regimes excluding patients with advanced cognitive impairment, and the need to recruit a study partner or companion alongside a patient for when the disease progresses. While patient burden must always be balanced with safety monitoring, the age of the $\mathrm{AD}$ population and the presence of co-morbidities can make this particularly challenging $[5,6]$.

Although a recent Cochrane review identified 68 studies evaluating strategies to improve RCT recruitment, none of these strategies had been tested in $\mathrm{AD}$ populations [7], and there is a paucity of empirical evidence supporting trial recruitment in this clinical area [5,6]. Qualitative research can improve trial recruitment in some cases, [8,9] but has not yet been applied to an AD trial [10-13]. This study aimed to identify potential challenges to, and facilitators of, recruitment to the Reducing pathology in Alzheimer's Disease through Angiotensin TaRgeting (RADAR) RCT, and to draw out implications for improving recruitment to AD trials more widely [14].

\section{METHODS}

Semi-structured telephone interviews were conducted with clinicians and trial staff recruiting to the RADAR trial, a phase II multi-center RCT comparing effects of $100 \mathrm{mg}$ losartan with placebo on changes in brain whole atrophy in patients with $\mathrm{AD}$ (Trial Registration Number ISRCTN93682878) [14]. Inclusion criteria were diagnosis with mild-moderate AD and at least 55 years old. The trial took place in a total of 23 sites which were specialist UK National Health Service (NHS) hospital trusts that routinely diagnose AD. Participants enrolled in the trial underwent an initial open label phase. If successful, they were randomized to either losartan or placebo for 12 months. Patients and trial personnel were blinded to the treatment arms for trial duration. The primary outcome, rate of whole brain atrophy as a surrogate measure of disease progression, was assessed through a magnetic resonance imaging (MRI) scan at 12 months. Recruitment for the trial took place between July 2014 and May 2018. The recruitment target was 228 and final recruitment was 211 patients $(92.5 \%)$. Initially 10 sites were to be included in the trial, but low recruitment led to more sites being added and the timeline for the trial being extended.

\section{Sampling}

Interview participants were purposively sampled to include research nurses and doctors screening and consenting patients, from trial sites with high and low recruitment rates compared to targets. The target recruitment rate per site was 0.4 patients per month $; \geq 0.4$ was categorized as a high recruitment rate and $<0.39$ as low.

\section{Data collection}

Semi-structured telephone interviews were conducted by CC, LES, and JH, experienced qualitative researchers, independent of the trial team. Participants were unknown to the interviewers beforehand. Participants were informed of the study aims and purpose of the interviews. A semi-structured topic guide (Box 1) was used to assist questioning during the interview while allowing participants to introduce unanticipated issues. Data collection continued until theoretical saturation, i.e., later stages of analysis resulted in no new themes being identified [15]. Interviews were conducted between July and October 2016 when the trial had been recruiting for 23 months.

Box 1. Summary of topic guide
Interview topics for discussion:
The site recruitment pathway
Views on and examples of explaining the trial details to patients;
- Randomization
- Uncertainty and informed consent
Patient views of the trial and potential preferences regarding trial arm allocation
Positive and negative aspects of recruitment
Recommendations for improving recruitment to future AD trials.


Table 1

Thematic summary of barriers (-) and facilitators (+) to recruitment

\begin{tabular}{|c|c|c|}
\hline Theme & Barriers (-) & Facilitators (+) \\
\hline 1. System & $\begin{array}{l}\text { AD diagnostic pathway } \\
\text { poor record keeping and quality of information } \\
\quad \text { (local) } \\
\text { research not embedded in culture of AD care } \\
\text { lack of/inaccurate information on national database } \\
\text { resources needed to use the national database } \\
\text { self-selected nature of patients on national database }\end{array}$ & $\begin{array}{l}\text { linking up systems with other sites } \\
\text { establish links with other services and recruitment } \\
\text { avenues } \\
\text { make research part of standard treatment options } \\
\text { multiple staff members using national database }\end{array}$ \\
\hline $\begin{array}{l}\text { 2. Healthcare } \\
\text { Professionals }\end{array}$ & $\begin{array}{l}\text { gate-keeping } \\
\text { lack of connection with other sites }\end{array}$ & $\begin{array}{l}\text { personal knowledge/direct contact } \\
\text { good links with other researchers/sites } \\
\text { staff training }\end{array}$ \\
\hline $\begin{array}{l}\text { 3. Patient and } \\
\text { companions }\end{array}$ & $\begin{array}{l}\text { requirement for research partner/companion } \\
\text { research partner/companion acting as a gate-keeper } \\
\text { overly complex/unnecessarily detailed } \\
\text { language/materials } \\
\text { insufficient information on key topics } \\
\text { concerns for clinical condition and prolonged } \\
\text { participation } \\
\text { preference for active arm }\end{array}$ & $\begin{array}{l}\text { presentation of clear, simple and concise information } \\
\text { follow-up with patient } \\
\text { prior patient involvement in research } \\
\text { patient belief in the trial and its benefits }\end{array}$ \\
\hline
\end{tabular}

\section{Data analysis}

All interviews were audio recorded, professionally transcribed verbatim, imported into NVivo 10 [16] and analyzed using thematic analysis [17] and constant comparison techniques [18, 19]. CC, LES, and $\mathrm{JH}$ independently coded three transcripts to establish initial coding frames. Coding was both inductive, identifying patterns and themes in the data which addressed important issues for participants, and deductive, focused on trial recruitment [20]. Analysts then met to discuss emerging findings and agree a final coding frame. CC and LES applied the final coding frame to all transcripts. Themes and subthemes were compared within and across interviews and sites to identify patterns and confirming and disconfirming instances. Our research was in the subtle realist paradigm, in which reality is conceptualized as existing objectively, but known only from each individual's own perspective.

\section{Ethics, consent, and permissions}

All procedures contributing to this work comply with the ethical standards of the relevant national and institutional committees on human experimentation and with the Helsinki Declaration of 1975, as revised in 2008. All procedures involving human subjects/patients were approved by NHS Research Ethics Committee (12/WA/0338). All participants gave informed consent and agreed to anonymized quotes being used in publications. In presenting findings, data have been anonymized to protect confidentiality. Data extracts are presented by trial site number, role of interviewee and whether the site was high or low recruiting (e.g., Site 2, Doctor, high recruiting).

\section{RESULTS}

Interviews were conducted with 17 healthcare professional participants. Seven were doctors and ten nurses who were either research nurses or research coordinators involved in patient recruitment. Participants were sampled from 10 of the 23 trial sites across the UK, 5 sites were high recruiting (8 participants interviewed), and 5 sites were low recruiting ( 9 participants interviewed). Interviews lasted between 11 and 90 minutes (mean 36 minutes).

Challenges to and facilitators of recruitment related to three themes: 1) Systemic factors (AD diagnostic pathway, patient records, embedding research in patient care, and the national research database), 2) Healthcare professionals, and 3) Patients and their companions (see Table 1 for summary).

\section{Theme 1: Systemic factors}

System-level challenges and facilitators occurred in four main sub-themes: the diagnostic pathway, patient records, embedding research in patient care, and the national research database. 


\section{AD diagnostic pathway}

Interviewees reported that patients with $\mathrm{AD}$ are currently most often diagnosed later in the disease, and most mild-moderate AD patients go undetected. This made it difficult to identify patients with mildmoderate $\mathrm{AD}$ who might be eligible for the trial.

"It's just working in a system where it's hard to find people where they're not diagnosed until late... That's probably the main [difficulty]: just finding the right people, so someone that's had a diagnosis of $[\mathrm{AD}]$ and they're in that mild to moderate category so, yes that can be challenging." (Site 2, Doctor, high recruitment)

Interviewees also reported that $\mathrm{AD}$ was being increasingly diagnosed in primary care, and that patients with mild-moderate $\mathrm{AD}$ therefore did not necessarily access the services, such as memory clinics, where the trial was recruiting.

"[The consultant] doesn't necessarily get an awful lot of patients through the clinic that would be eligible for us ... often patients aren't referred to a memory clinic [by their GP]. People cope with the dementia without asking for help for a long time... They don't get an awful lot of follow-up once they're diagnosed as well." (Site 4 , Nurse, high recruiting)

The specialist nature of some AD clinics meant the patient pool was even more limited.

"That's a relatively small pool of people because our clinics are tertiary referral clinics, so we tend to get some people with the unusual dementias rather than very standard Alzheimer's disease." (Site 2, Doctor, high recruiting)

To address this, several higher recruiting sites had established links with primary care and engaged with a wide range of services and recruitment avenues outside the clinic. These included using the national Join Dementia Research (JDR) database, [21] community memory services, specialist and research nurses, as well as advertisements and media publicity.

"We also have good links with the psychiatric nurses in [area] and they followed us on you know, they might see patients on their caseload, they typically will come back to us and say, 'You know, so-and-so has been tested and is eligible' and then we might pursue a potential participant that way as well." (Site 3, Nurse, high recruiting)
Linking with and using additional recruitment avenues required understanding, engagement, and planning with local services early on in trial development.

"It's just linking with the right clinics and clinicians or GPs, wherever they're being diagnosed, so then that has its challenges because everywhere's doing it differently in the UK ... there's lots of different streams and systems. So, it's just getting your head around who does what, who's gonna have communication with these patients and then making sure you've got all the right agreements in place. That can be quite a lot to get your head around." (Site 2, Doctor, high recruiting)

\section{Patient records}

Interviewees relied on patient medical records and local research databases for pre-screening, so poor quality record-keeping or IT access problems hindered the identification of eligible patients.

"Our electronic records may not document what medication they're on at the moment, because our system is a mental health system and it doesn't link up with the GP system... so we probably haven't got their current up-to-date medication so, yeah, that can be quite difficult". (Site 5, Nurse, low recruiting)

Local research databases of patients who had agreed to be approached about relevant research studies were rarely regularly updated, which resulted in staff contacting patients who were ineligible.

"We also had a database that we went through, but the database is quite old, so a lot of patients were too old or too unwell to take part when they were given the opportunity." (Site 4, Nurse, high recruiting)

In contrast, one interviewee at a high recruiting site discussed how linking up systems with other sites aided patient identification and screening:

"Our system is quite good in [area] 'cause there's a lot of notes that have been scanned in from other hospitals so you can actually view them. It depends on how local the hospitals are, but they've also got a scan store thing, so [if] they've had like an MRI or CT [computerised tomography] scan in another health board, you can look that up on the [system] as long as you have 
their identification number." (Site 4, Nurse, high recruiting)

\section{Embedding research in patient care}

Research not being embedded in the culture of AD care across services contributed to difficulties identifying and recruiting participants.

'It's more bedding the research into the culture... because when people are given a diagnosis of dementia there's a huge emphasis on living well, accepting it and not having unrealistic expectations, coping with the emotions of it... I think research doesn't get mentioned.' (Site 2, Doctor, high recruiting)

To address this, staff at high recruiting sites asked all patients for consent to be contacted about future research on admission to the service. This facilitated easier identification and recruitment and helped ensure all patients had access to research options.

"So as part of the clinic we automatically obtain consent or not... to be contacted about studies... what's certainly pretty good is that people are happy to be consented to be contacted. So that's been for ... a number of years... part of the routine procedure in clinic, to have this consent in place”. (Site 6, Doctor, high recruiting)

\section{National research database}

Most staff at high recruiting sites discussed using the JDR national database for trial patient identification. People with dementia and their carers can sign up online to join the JDR and register their interest in taking part in dementia research. Staff at lower recruiting sites discussed using JDR but not as actively as the higher recruiting sites; they adopted a more "passive approach" (Site 9, Nurse, low recruiting) and extra resources were needed to use it effectively.

All those who used JDR discussed the same challenges using the database: it lacked clarity on patients' exact diagnosis, progression status and capacity, and information on patients' participation in other research studies was often not current. Interviewees felt JDR needed to be updated regularly to remain relevant and useful. Most patients identified through JDR were unsuitable.

"The Join Dementia Research website keeps me very busy [laugh]... I think we've recruited one person from there, but there's always lots of people to look at. Whether they are suitable is another thing." (Site 2, Nurse, high recruiting)

Owing to the incompleteness of the JDR, trial staff had to spend considerable time contacting potential participants to discuss missing information and gain their consent to seek out additional information before they could reliably identify them as trialeligible.

Interviewees had only limited time within their busy workload to dedicate to the trial. They suggested that having multiple members of the trial team screening and approaching patients from JDR would benefit recruitment.

"Just having more than one person pre-screening so you've always for people following up people... I think that does take up a lot of time... it does help if you've got two or three of you." (Site 2, Nurse, high recruiting)

\section{Theme 2: Healthcare professionals}

The clinicians interviewed expressed positive views of the trial purpose, design, and leadership which led them to want to be involved.

"There are no treatments for dementia or Alzheimer's disease. [Losartan] is a very cheap, easy medication to give that we already know is very safe and there's a good scientific rationale so, you know, even if there was any benefit at all from it [Lorsatan] would be a big step up for people." (Site 2, Doctor, high recruiting)

Clinicians preferred to approach patients from their own clinics for trial recruitment, "because you know them" (Site 3, Doctor, high recruiting), and assessing eligibility for the trial through known medical history was straight-forward.

"Well, the ones who we have from a PI [Principal Investigator]'s clinic, you will already be aware of their medical history and things, which is probably why that's the most successful recruitment method up here, because we're aware of the medical history against the inclusion/exclusion criteria." (Site 3, Nurse, high recruiting)

However, interviewees described some clinicians being paternalistic with their own patients and acting as gate-keepers, restricting the number of patients they approached about a trial. 
"A lot of clinicians are very reluctant to quote 'allow' unquote their patients to go into research." (Site 3, Doctor, high recruiting)

Few interviewees had undertaken any "formal training" (Site 6, Doctor, high recruiting) in recruitment to trials, and most relied on previous experience and learning on the job. Some interviewees identified issues with explaining the trial to patients, particularly randomization and the necessity for a placebo arm, and found it "hard to manage people's expectations." (Site 2, Nurse, high recruiting). They felt they would benefit from training on how to deal with these issues.

"I think sometimes at the end it's sort of blustering around a bit and not feeling confident... that's not robust and I think we need to remember that when we express it to our patients ... I think that would be a useful thing to have some sort of training and ... based on evidence in how we express trials to patients, how we convey the need for placebo and the need for the rigour." (Site 2, Doctor, high recruiting)

Interviewees reported that having a platform to communicate with other sites (e.g., newsletters and joint conference calls) could help them discuss successes and challenges and allow them to learn from each other. This would, they believed, help increase recruitment at sites.

"We could see... which site is really recruiting well and get in touch with them and say, like, 'What are you doing to recruit really well? Is there something that we can do?"' (Site 4, Nurse, low recruiting)

\section{Theme 3: Patients and their companions}

One of the main challenges to patients taking part in the trial was the eligibility requirement of having a research companion (an informant) support them through the trial; many of the patients approached did not have an appropriate person or did not want to burden a family member or friend. The number of research visits, held during working hours, made this more challenging.

"Another criteria that does exclude a few people actually is they have to have someone who we call an informant who will come with them to certain visits, so I think it's the screening, baseline, six months and twelve month visits they would have to come with someone, and actually quite a lot of people live on their own and then they feel like, oh, 'I can't ask my son or daughter because they'll be too busy,' or 'I don't really want my friends to come with me'. Because we do ask the informant quite a lot of questions about the participant, about their memory and how they are and how they seem, so that can exclude quite a lot of people as well." (Site 2, Doctor, high recruiting)

Potential research companions were sometimes gatekeepers to patient recruitment, and managing both patients' and companions' expectations of the trial could be challenging for staff.

"Well, it's usually the informant to be honest... Occasionally the individual will say, 'No, I don't think I'm up to that.' But usually it's [the] companion, 'I don't think they'll be able to do it,' you know." (Site 3, Doctor, high recruiting)

Patients themselves could potentially be deterred by the trial participant information sheets, which were perceived as lengthy and complicated.

"Once we do invite them to the screening visit, we then have to send another patient information sheet, patient information sheet 2 and then another information sheet for the informant as well and that's quite a lot of paperwork... to send. So I think some people when they get that are a bit like, 'Wow! There's a lot of information to take in."' (Site 2, Nurse, high recruiting)

Patients and their informants commonly asked for clarification of the written information. Most queries related to the number and location of research visits, drug side effects, monitoring, what would happen once the study ended, and whether they would be informed about study results.

"[Patients] want to have more [reassurance] around the fact that... they don't have to come to sites for all their visits ... and they typically want to ask maybe a few more questions about the drug." (Site 3, Nurse, high recruiting)

"Usually people ask about side-effects." (Site 6, Doctor, high recruiting)

Interviewees highlighted the need to stagger and reiterate information and the benefit of multiple opportunities to discuss the trial, either in clinic or by phone. 
"I try and make it as simple as possible... we give them opportunities to ask more questions. I try not to use words that they may not understand. I try and simplify the discussion as much as possible." (Site 9, Doctor, low recruiting)

"We normally telephone them just because we find that works best, [rather] than just sending them out information and hoping that they will read and understand it." (Site 3, Nurse, high recruiting)

The length of the study and the treatment meant patients feared they would not be able to last the duration; they felt they would be too unwell or too fragile or that they might not be able to take the medication as required.

"The main reasons [for not taking part] really are [that] ... they feel their own health might not last for a year, or things are a bit fragile at home for instance, you know ... or they might not be able to guarantee that they would take the medication for a year." (Site 3, Doctor, high recruiting)

Interviewees described most patients being happy with the possibility they may be allocated to the placebo arm. However, some interviewees had experienced people declining or withdrawing participation for this reason.

"If she was on placebo, they [patient and companion] would probably not continue the trial... and then they did feel she was on placebo, so they didn't continue the trial." (Site 2, Doctor, high recruiting)

Patients' and companions' positive views of the trial and what it was trying to achieve facilitated participation. They saw the trial as potentially benefitting themselves and others in the future.

"I mean some of them would hope that they had some benefit from the trial, even if it's just to be looked after more carefully and... I mean they don't know if they are going to have benefit or not but at least the trial is going to be useful to future generations." (Site 4, Nurse, high recruiting)

Patients and their companions also liked that the trial was run by a University, with no financial interest in the outcome.

"Some quite appreciate the fact, you know, that they're taking part in a university-led trial... there are some participants who just don't support the whole commercial kind of thing, you know the big money-making organisation and they do quite like the fact that it's a university associated trial." (Site 3, Nurse, high recruiting)

\section{DISCUSSION}

\section{Main findings}

This study provides an in-depth and novel examination of the views and experiences of those involved in recruiting to an $\mathrm{AD}$ trial, sampled from a range of high and low recruiting sites. Barriers to trial recruitment included systemic factors as well as factors related to healthcare professionals, patients, and their companions, and are likely to have relevance to other AD trials. Current diagnostic pathways and data systems and poor-quality record-keeping made identifying and screening patients difficult. Routinely embedding research into patient care helped facilitate recruitment. Clinicians preferred to recruit from their own clinics, but this could result in gatekeeping and restricted access for potentially eligible patients. Interviewees lacked formal recruitment training and believed that communication with other recruiters could help facilitate learning and improve recruitment. The study's requirement of a research companion was a major challenge, compounded by the timing and frequency of trial visits. Participants were put off trial participation by lengthy and complicated trial information and often had queries regarding key aspects of the trial. Given the progressive nature of $\mathrm{AD}$, patients were wary of taking part in a trial with a year of treatment and follow-up. Potential participants having a positive view of the trial, who was running it, and what it was trying to achieve helped facilitate recruitment.

\section{Findings within the context of wider literature}

Our results are consistent with previous studies highlighting that patient burden and a requirement for a study partner impact willingness to take part in an AD trial [6]. Grill and Karlawish [5] highlighted that traditional clinic visits taking place during the working day could restrict caregivers acting as study partners and therefore prevent patients participating. Our findings are also consistent with those of Watson and colleagues [6], who report a lack of $\mathrm{AD}$ diagnostic tools in primary care limiting identification and trial recruitment. We found that inadequate explanation of trial processes such as randomization, 
resulting in lack of patient understanding, impacted recruitment. This is consistent with recruitment barriers well-documented in non-AD trials [1]. The extent to which research is embedded in AD care has also been highlighted as a challenge to dementia research participation, with a recommendation that physicians routinely discuss referral to dementia research with their patients [22]. We found additional challenges in recruitment related to record-keeping and access to accurate patient information; lack of recruitment training and linkage between trial staff; and long, complex information sheets. Gatekeeping by healthcare professionals is a problem in other types of trials [23] where there is lack of equipoise; however, our findings indicate that the perceived vulnerability of patients with $\mathrm{AD}$ may further compound the problem, restricting opportunities for patients to participate. Our findings also highlight the influence of who is running the trial on patients' willingness to take part.

\section{Strengths and weaknesses}

The study included a range of recruiting staff at trial sites with differing recruitment rates. This study also benefits from a focus on mild-moderate AD, which previous studies have not addressed in depth. However, we did not capture patients' or their companions' voices, and this should be considered when interpreting our findings. It may be that in exploring their perspective, additional insights and therefore enhanced recommendations could be made.

\section{Implications for trials in $A D$}

Trial designs need to take into account the difficulty of identifying eligible participants by having pragmatic trial entry criteria and realistic recruitment projections. To facilitate recruitment, participant information should only contain essential information and be designed with input from Patient and Public Involvement (PPI) contributors, while adhering to research governance requirements. The timing of participant and companion visits should be flexible to improve convenience and kept to a minimum to reduce burden. Site staff should be provided with recruitment training, and research nurses and clinicians linked via newsletters and teleconference calls to enhance communication, training and site enthusiasm.

On the basis of our findings, we recommend that 1) steps are taken to improve diagnostic services and the way information about $\mathrm{AD}$ patients is recorded and shared, 2) opportunities to participate in research are routinely embedded into standard patient treatment/care pathways, 3) there is less reliance on individual clinicians, services and databases for recruitment, and increased linkage and use of a wide range of services and approaches to identify and recruit patients, 4) trial teams capitalize on enthusiasm for $\mathrm{AD}$ trials; for example, through sharing patient stories or testimonials.

\section{ACKNOWLEDGMENTS}

This project is funded by the Efficacy and Mechanism Evaluation (EME) Programme, an MRC and NIHR partnership (EME- 11/47/03). This study was designed and delivered in collaboration with the Bristol Randomised Trials Collaboration (BRTC), a UKCRC registered clinical trials unit which, as part of the Bristol Trials Centre, is in receipt of National Institute for Health Research CTU support funding.

The views expressed in this publication are those of the author(s) and not necessarily those of the MRC, NIHR, or the Department of Health and Social Care.

*The EME Programme is funded by the MRC and NIHR, with contributions from the CSO in Scotland and Health and Care Research Wales and the HSC R\&D Division, Public Health Agency in Northern Ireland.

The authors thank the staff who took part in the interviews and the invaluable support of the RADAR trial research team.

Authors' disclosures available online (https:// www.j-alz.com/manuscript-disclosures/19-0146r1).

\section{REFERENCES}

[1] Maughan TS, Meade AM, Adams RA, Richman SD, Butler R, Fisher D, Wilson RH, Jasani B, Taylor GR, Williams GT, Sampson JR, Seymour MT, Nichols LL, Kenny SL, Nelson A, Sampson CM, Hodgkinson E, Bridgewater JA, Furniss DL, Roy R, Pope MJ, Pope JK, Parmar M, Quirke P, Kaplan R (2014) A feasibility study testing four hypotheses with phase II outcomes in advanced colorectal cancer (MRC FOCUS3): A model for randomised controlled trials in the era of personalised medicine? Br J Cancer 110, 2178-2186.

[2] Nelson A, Fenlon D, Morris J, Sampson C, Harrop E, Murray N, Wheatley D, Hood K, Griffiths G, Barrett-Lee P (2013) QUALZICE: A QUALitative exploration of the experiences of the participants from the ZICE clinical trial (metastatic breast cancer) receiving intravenous or oral bisphosphonates. Trials 14, 325 .

[3] Smith JD, Baillie J, Baglin T, Griffiths GO, Casbard A, Cohen D, Fitzmaurice DA, Hood K, Rose P, Cohen AT, Johnson M, Maraveyas A, Bell J, Toone H, Nelson A, Noble SI (2014) A feasibility study to inform the design 
of a randomized controlled trial to identify the most clinically and cost effective Anticoagulation Length with low molecular weight heparin In the treatment of Cancer Associated Thrombosis (ALICAT): Study protocol for a mixed-methods study. Trials 15, 122.

[4] Harrop E, Kelly J, Griffiths G, Casbard A, Nelson A (2016) Why do patients decline surgical trials? Findings from a qualitative interview study embedded in the Cancer Research UK BOLERO trial (Bladder cancer: Open versus Lapararoscopic or RObotic cystectomy). Trials $\mathbf{1 7}, 35$.

[5] Grill JD, Karlawish J (2010) Addressing the challenges to successful recruitment and retention in Alzheimer's disease clinical trials. Alzheimers Res Ther 2, 34.

[6] Watson JL, Ryan L, Silverberg N, Cahan V, Bernard MA (2014) Obstacles and opportunities in Alzheimer's clinical trial recruitment. Health Aff (Millwood) 33, 574-579.

[7] Treweek S, Pitkethly M, Cook J, Fraser C, Mitchell E, Sullivan F, Jackson C, Taskila TK, Gardner H (2018) Strategies to improve recruitment to randomised trials. Cochrane Database Syst Rev 2, Mr000013.

[8] Donovan JL, Lane JA, Peters TJ, Brindle L, Salter E, Gillatt D, Powell P, Bollina P, Neal DE, Hamdy FC (2009) Development of a complex intervention improved randomization and informed consent in a randomized controlled trial. $J$ Clin Epidemiol 62, 29-36.

[9] Mills N, Donovan JL, Wade J, Hamdy FC, Neal DE, Lane JA (2011) Exploring treatment preferences facilitated recruitment to randomized controlled trials. J Clin Epidemiol 64, 1127-1136.

[10] Wade J, Donovan JL, Lane JA, Neal DE, Hamdy FC (2009) It's not just what you say, it's also how you say it: Opening the 'black box' of informed consent appointments in randomised controlled trials. Soc Sci Med 68, 2018-2028.

[11] Donovan JL, Rooshenas L, Jepson M, Elliott D, Wade J, Avery K, Mills N, Wilson C, Paramasivan S, Blazeby JM (2016) Optimising recruitment and informed consent in randomised controlled trials: The development and implementation of the Quintet Recruitment Intervention (QRI). Trials 17, 283.

[12] Paramasivan S, Huddart R, Hall E, Lewis R, Birtle A, Donovan JL (2011) Key issues in recruitment to randomised controlled trials with very different interventions: A qualitative investigation of recruitment to the SPARE trial (CRUK/07/011). Trials 12, 78-78.

[13] Horwood J, Johnson E, Gooberman-Hill R (2016) Understanding involvement in surgical orthopaedic randomized controlled trials: A qualitative study of patient and health professional views and experiences. Int J Orthop Trauma Nurs 20, 3-12.

[14] Kehoe PG, Blair PS, Howden B, Thomas DL, Malone IB, Horwood J, Clement C, Selman LE, Baber H, Lane A, Coulthard E, Passmore AP, Fox NC, Wilkinson IB, BenShlomo Y (2018) The Rationale and Design of the Reducing Pathology in Alzheimer's Disease through Angiotensin TaRgeting (RADAR) Trial. J Alzheimers Dis 61, 803-814.

[15] Greg G, Arwen B, Laura J (2006) How many interviews are enough?: An experiment with data saturation and variability. Field Methods 18, 59-82.

[16] QSR International, What is NVivo? | NVivo, https://www.qsrinternational.com/nvivo/what-is-nvivo

[17] Braun V, Clarke V (2006) Using thematic analysis in psychology. Qual Res Psychol 3, 77-101.

[18] Glaser BG, Strauss AL (2009) The Discovery of Grounded Theory: Strategies for Qualitative Research, Aldine Transaction.

[19] Charmaz K (2014) Constructing Grounded Theory, SAGE Publications.

[20] Boyatzis RE (1998) Transforming Qualitative Information: Thematic Analysis and Code Development, SAGE Publications.

[21] Join Dementia Research, Join dementia research register your interest in dementia research : Home, https://www.joindementiaresearch.nihr.ac.uk/

[22] Global Action Against Dementia by Alzheimer's Disease International Member Charities (2014) Participation in dementia trials and studies: Challenges and recommendations.

[23] Potter S, Mills N, Cawthorn SJ, Donovan J, Blazeby JM (2014) Time to be BRAVE: Is educating surgeons the key to unlocking the potential of randomised clinical trials in surgery? A qualitative study. Trials $\mathbf{1 5}, 80$. 